\title{
Interfacial Instability and Mixing in Stratified Flows
}

\author{
By Garbis H. Keulegan
}

\begin{abstract}
When a liquid flows over a body of heavier liquid, with which it is miscible, an interface may be formed at which there is a sharp discontinuity of densities but not necessarily of velocities. As the relative velocity is increased, waves are formed at the interface, and at a critical velocity, mixing begins by the formation of eddies periodically ejected from the crests of the waves. The numerical values of a criterion for mixing, whose form was derived from theoretical considerations, were determined experimentally. Experimental data were also obtained on the relation of the amount of mixing to the relative velocity of the liquids at velocities higher than the critical.
\end{abstract}

\section{Introduction}

The intrusion of salt water in rivers that communicate with the seas and the silting of reservoirs receiving muddy waters are the two most important examples of stratified flow where the question of interfacial behavior assumes a basic significance. Depending on the conditions of approach, two possible forms of the interface may be noted. In one form, the interface may be identified as the dividing surface of two layers of liquid with different densities, the surface being one of sharp discontinuity of densities but not necessarily of velocities. Ordinarily the interface of this type is the locale of internal waves if the difference in velocities at points on opposite sides of the interface and at some distance from it is large. If mixing is present, it is in the form of eddies that are periodically ejected from the crests of the waves into the current that has the greater velocity. In the other form, the interface is a layer of transition between two currents. Both the densities and the velocities change uniformly in the layer that has measurable thickness. If any mixing is present, it is associated with the momentum exchange of turbulence, and the regular pattern of internal waves is absent. Thus a study of interfaces may be confined to one or the other of these forms.

In the present investigation, which is restricted to an interface with sharp discontinuity of densities, one may visualize the following distinct problems. First, what quantities are to be considered in determining a criterion for the incidence of mixing? Second, with current velocities exceeding the critical velocity of the criterion, how is the amount of mixing related to the velocity? Third, what is the exact mechanism of flow that produces the mixing?

About a decade or so ago at the National Hydraulic Laboratory, experiments were carried out to study each of these three questions. After having studied the first and the second of them with some degree of satisfaction with the results obtained, we were about ready to enter into the study of the third, which obviously is beset with considerable experimental difficulties and theoretical uncertainties. Unfortunately at that time it became necessary to suspend the hydraulic work in the laboratory, and since then we have had no opportunity to return to our original plans. Meanwhile considerable interest in these matters has developed. Since numerous inquiries have been received about the progress already made, it was decided to make these results known notwithstanding their incompleteness.

\section{Determination of the Critical Velocity of Mixing}

Observations on the instability of interfaces were made for the condition where the upper, less dense liquid was flowing over a heavier liquid at rest in a pool. The essentials of the apparatus 
and the arrangements are shown in figure 1. The apparatus consisted of a closed flume with vertical glass walls and was limited at the entrance by a constant level tank and at the exit by a discharge tank. The large reservoir of the circulating liquid, which was the lighter liquid, was connected to the constant level tank through a pump. To
The flowing current caused the lower liquid to be dragged out in the downstream direction. In the small and the medium flumes, the escape of the lower liquid was prevented by tilting the flumes by means of screw-jack supports. This method was not practicable for the large flume. Instead, the flume was fixed in a moderately

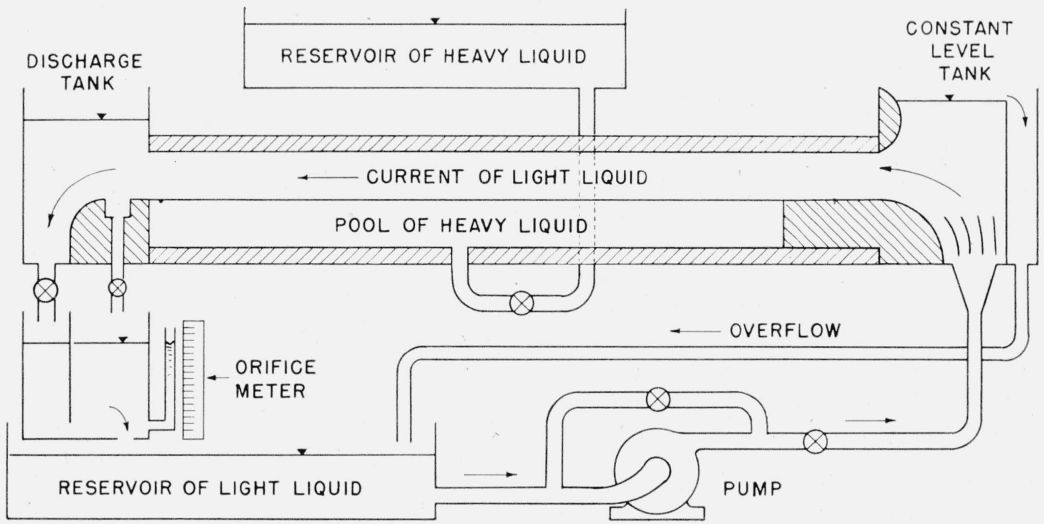

FIGURE 1. Diagram of flume and accessories.

prevent the transmission of pump vibrations to the flume frame, the connection to the constant level tank was made through a flexible rubber tubing. The return flow passed from the discharge tank into a metering tank of the orifice type, which in turn discharged its contents continually into the reservoir of light liquid below. The volume of the circulating liquid during a test remained the same, and since the reservoir volume was many times larger than the volume of the flume, mixing in the flume during a run had very little effect on the density of the upper liquid. The loss of the heavier liquid from the pool during tests was made up at intervals by drawing from a reservoir placed at a higher elevation.

For the purpose of examining the effect of Reynolds number, three flumes of various sizes were employed. They were rectangular in cross section, with the greater dimension vertical, with cross sectional dimensions of 2 by $4 \mathrm{~cm}, 4$ by 8 $\mathrm{cm}$, and 11.3 by $28.5 \mathrm{~cm}$. The length in each case was about 25 times the total depth. The flumes were operated full, keeping the depths of the two layers equal or nearly equal. Accordingly, the hydraulic radii associated with the currents were $0.50,1.00$, and $3.27 \mathrm{~cm}$. The flumes will be referred to as the small, the medium, and the large flume. inclined position. When the velocities of the current were too high, the lower liquid that was dragged out was prevented from entering the lower reservoir by drawing it off through a trap at the exit end. The idea involved in the method is shown in figure 2 .

The determination of the critical velocity; that is, the velocity at which the mixing of the liquid of the lower layer with the upper commenced, or was about to commence, was done visually by noting the condition of the interface. At low velocities a smooth interface was discernible even when the lower liquid was not colored. At this

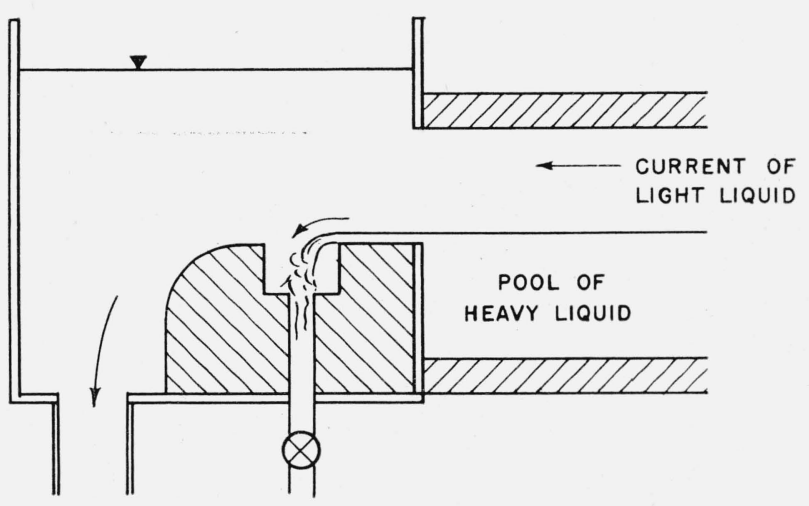

Figure 2. Diagram of diverter for heavy liquid dragged out of flume by the current of light liquid. 
initial stage, observations showed that at the interface, and in a finite band on the two sides of the interface, the flow was laminar even when the central regions of the upper current were eddying or turbulent. When the velocity of the current was increased to some definite value depending on the densities and the viscosities of the two liquids, the interface appeared to be covered with ridges extending from one glass wall to the other. These ridges moved progressively downstream, and their crest lines were parabolic in shape, the crest heights being greatest at the center. With a slight increase in the value of the current velecity, waves appeared in a new configuration. The interface now was covered with waves of shorter crest lines. In the tests with the large flume these crest lines were one-half or one-third of the channel width. The appearance of the waves and their sequence was regular. The movement was progressive. The predominant wavelength remained constant, or nearly so, throughout the channel length. With further increase of the velocity of the current, the waves became sharp-crested, and mixing commenced. At the beginning the mixing was slight, and it augmented with subsequent increases in velocity. It appeared that increased velocities did not substantially affect the wavelengths of the interfacial waves, although there was a tendency for the lengths to decrease slightly. The most marked influence of the increasing velocities was to augment the height of the sharpcrested waves, the size of the eddies emanating from the crests, and the frequency of departure of the eddies. Practically, the mechanism of mixing was an ejection of eddies from the crests into the moving current, as shown diagrammatically in figure 3.

Now, what constitutes the critical velocity of mixing is a matter difficult to decide. The method that we employed is as follows: In the record book was entered a description of the appearance of the interface, together with a qualitative statement regarding the degree of mixing for a measured velocity of the current. The interface, for example, was described as being smooth, or having parabolic waves, or mixed waves, or sharp crests, or agitated crests. The mixing was described as absent, or slight, or moderate, or excessive. We note the velocity $U_{0}$, the maximum velocity recorded in the book as corresponding to a smooth inter- face and to no mixing. Next we take the velocity $U_{1}$, which is the average of all the velocities associated with slight mixing. The mean value of $U_{0}$ and $U_{1}$ is chosen to represent the critical velocity of mixing. It is denoted by $U_{\mathrm{c}}$. The average value of the velocities associated with moderate mixing are denoted by $U_{2}$, and the average value above which there was always excessive mixing as $U_{3}$.

The test liquids were tap water and sugar solutions. Four series of tests were made. In three of the series the upper liquid consisted of tap water, and tests were made in all three flumes. The purpose of these tests was to determine the effect of the Reynolds number of the upper current on the incidence of mixing. The fourth series of tests was intended to determine the effect of viscosity on mixing. These tests were made with the medi-

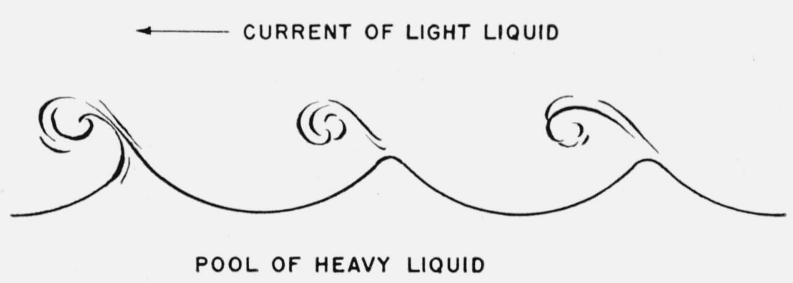

Figure 3. Diagram of process of mixing.

um flume. Both the upper liquid and the lower liquid were sugar solutions. The fractional difference of the densities was kept practically constant, but the viscosity of the upper liquid was varied. The velocity data from the tests of the four series are given in tables $1,2,3$, and 4 .

TABLE 1. Mixing tests in the small flume (Hydraulic radius $=0.50 \mathrm{~cm}$ )

\begin{tabular}{|c|c|c|c|c|c|c|c|c|c|}
\hline Run & $\rho_{1}$ & $\rho_{2}$ & $\nu_{1}$ & $\nu_{2}$ & $U_{c}$ & $U_{0}$ & $U_{1}$ & $U_{2}$ & $U_{3}$ \\
\hline & $\mathrm{g} / \mathrm{cm}^{3}$ & $\mathrm{~g} / \mathrm{cm}^{3}$ & $\mathrm{~cm}^{2} / \mathrm{sec}$ & $\mathrm{cm}^{2} / \mathrm{sec}$ & $\mathrm{cm} / \mathrm{sec}$ & $\mathrm{cm} / \mathrm{sec}$ & $\mathrm{cm} / \mathrm{sec}$ & $\mathrm{cm} / \mathrm{sec}$ & $\mathrm{cm} / \mathrm{sec}$ \\
\hline C-13... & 1.000 & 1. 097 & 0.0086 & 0.0174 & 7.45 & 6.97 & 7.94 & 9. 96 & 13. 01 \\
\hline C-14.. & 1.000 & 1. 185 & .0086 & .0455 & 9.14 & 8.12 & 10.16 & 12.85 & 15. 28 \\
\hline C-15_.. & 1.000 & 1. 173 & .0085 & .0395 & 10.18 & 8.47 & 11.89 & 14.65 & 23.65 \\
\hline C-16... & 1.000 & 1. 149 & .0084 & .0283 & 7.99 & 7.00 & 8.98 & 12. 61 & 16. 53 \\
\hline C-17... & 1.000 & 1. 095 & .0080 & .0151 & 7. 12 & 6.34 & 7. 91 & 10.15 & 14.65 \\
\hline C-18_.. & 1.000 & 1. 064 & .0080 & .0120 & 6.98 & 5. 91 & 8.06 & 10.35 & 14. 24 \\
\hline C-19..- & 1.000 & 1. 061 & .0079 & .0119 & 6.19 & 5.58 & 6.81 & 9.16 & 12.56 \\
\hline $\mathrm{C}-20 \ldots$ & 1.000 & 1. 036 & .0082 & .0101 & 6.25 & 5.65 & 6.85 & 8.02 & 9. 63 \\
\hline C-21_.. & 1.000 & 1. 017 & .0081 & .0088 & 5. 21 & 4. 61 & 5. 82 & 6. 96 & 8.37 \\
\hline $\mathrm{C}-22 \ldots$ & 1.000 & 1.007 & .0082 & .0083 & 4.08 & 3.92 & 4. 27 & 5.01 & 5.41 \\
\hline C-23... & 1.000 & 1. 226 & .0081 & .0800 & 8.14 & 7. 96 & 9.52 & 14. 71 & 20.45 \\
\hline
\end{tabular}


TABLE 2. Mixing tests in the medium flum

(Hydraulic radius $=1.00 \mathrm{~cm}$ )

\begin{tabular}{|c|c|c|c|c|c|c|c|c|c|}
\hline Run & $\rho_{1}$ & $\rho_{2}$ & $\nu_{1}$ & $\nu_{2}$ & $U_{c}$ & $U_{0}$ & $U_{1}$ & $U_{2}$ & $U_{3}$ \\
\hline & $\mathrm{g} / \mathrm{cm}^{3}$ & $\mathrm{~g} / \mathrm{cm}^{2}$ & $\mathrm{~cm}^{2} / \mathrm{sec}$ & $\mathrm{cm}^{2} / \mathrm{sec}$ & $\mathrm{cm} / \mathrm{sec}$ & $\mathrm{cm} / \mathrm{sec}$ & $\mathrm{cm} / \mathrm{sec}$ & $\mathrm{cm} / \mathrm{sec}$ & $\mathrm{cm} / \mathrm{sec}$ \\
\hline D-1 $1 \ldots$ & 1.000 & 1. 038 & 0.0088 & 0.0131 & 4. 39 & 3.85 & 4. 93 & 5. 37 & 6. 63 \\
\hline D-2 . . & 1.000 & 1. 044 & .0095 & .0129 & 4. 59 & 3. 96 & 5. 23 & 5. 50 & 5. 81 \\
\hline D-3 & 1.005 & 1. 052 & .0095 & .0140 & 4.55 & 4. 47 & 4. 62 & 5. 02 & 5. 41 \\
\hline D-4 & 1. 026 & 1. 062 & .0106 & .0137 & 4. 75 & 4. 47 & 4. 62 & 5.02 & 5. 41 \\
\hline D-5 & 1.037 & 1.085 & 0118 & .0175 & 5.52 & 5. 05 & 5. 99 & 6.67 & 7. 62 \\
\hline D-6 ... & 1. 049 & 1. 094 & .0137 & .0204 & 6.49 & 6.07 & 6.91 & 7.45 & 8.60 \\
\hline D-7 & 1. 064 & 1. 103 & .0163 & .0235 & 6.88 & 6. 86 & 6.91 & 7.27 & 7. 71 \\
\hline D-8 & 1. 074 & 1. 119 & .0178 & .0277 & 7.18 & 6. 68 & 7. 68 & 8.03 & 8. 23 \\
\hline D-9 & 1. 088 & 1. 122 & .0192 & .0268 & 7. 04 & 6. 53 & 7.56 & 8.04 & 8.80 \\
\hline D-10 . & 1. 100 & 1. 139 & .0203 & .0301 & 6.97 & 6. 77 & 7.18 & 7. 60 & 8.75 \\
\hline D-11 & 1. 128 & 1. 164 & .0233 & .0351 & 7. 68 & 7. 65 & 7. 72 & 8. 20 & 8.00 \\
\hline D-12. & 1.146 & 1. 183 & .0276 & .0449 & 8.56 & 7. 96 & 9.17 & 9. 26 & 9. 31 \\
\hline D-13 & 1. 161 & 1. 203 & .0336 & .0591 & 9.98 & 9.31 & 10.66 & 11. 28 & 12.19 \\
\hline D-14 & 1. 171 & 1. 204 & .0393 & .0642 & 10.46 & 9.78 & 11.15 & 11.93 & 12. 60 \\
\hline D-15 & 1. 205 & 1. 245 & .0799 & .1563 & 13. 29 & 12. 17 & 14.41 & 14. 98 & 15. 46 \\
\hline D-16 & 1. 225 & 1. 257 & .1020 & .1970 & 13.17 & 11. 74 & 14. 61 & 15.80 & 16. 74 \\
\hline
\end{tabular}

TABLE 3. Mixing tests in the medium flume

$($ Hydraulic radius $=1.00 \mathrm{~cm})$

\begin{tabular}{|c|c|c|c|c|c|c|c|c|c|}
\hline Run & $\rho_{1}$ & $\rho_{2}$ & $\nu_{1}$ & $\nu_{2}$ & $U_{0}$ & $U_{0}$ & $U_{1}$ & $U_{2}$ & $U_{3}$ \\
\hline & $\mathrm{g} / \mathrm{cm}^{3}$ & $\mathrm{~g} / \mathrm{cm}^{3}$ & $\mathrm{~cm}^{2} / \mathrm{sec}$ & $\mathrm{cm}^{2} / \mathrm{sec}$ & $\mathrm{cm} / \mathrm{sec}$ & $\mathrm{cm} / \mathrm{sec}$ & $\mathrm{cm} / \mathrm{sec}$ & $\mathrm{cm} / \mathrm{sec}$ & $\mathrm{cm} / \mathrm{sec}$ \\
\hline D-18 & 1.000 & 1. 266 & 0.0085 & 0.1890 & 10.12 & 9. 12 & 11.13 & - & - \\
\hline D-19_. & 1.000 & 1. 139 & .0092 & .0318 & 7.86 & 6.44 & 9. 28 & 11.10 & - n..... \\
\hline D-20_- & 1.000 & 1. 135 & .0090 & .0264 & 8.31 & 6.02 & 10.60 & 13.50 & 15.57 \\
\hline D-21 & 1.000 & 1. 133 & .0094 & .0272 & 8.54 & 6.62 & 10.47 & 12. 14 & 13. 25 \\
\hline D-22 - & 1.000 & 1. 158 & .0094 & .0370 & 8.56 & 6.57 & 10.56 & 12.43 & 14.96 \\
\hline D-23_. & 1.000 & 1. 104 & .0094 & .0204 & 7. 30 & 5. 98 & 8. 62 & 10.83 & 12.06 \\
\hline D-24 & 1.000 & 1.094 & .0092 & .0180 & 6.55 & 5. 68 & 7. 42 & 9.92 & 12.03 \\
\hline D-25 & 1.000 & 1. 102 & .0092 & .0139 & 6.87 & 5. 74 & 8.00 & 9.89 & 11. 38 \\
\hline D-26_. & 1.000 & 1.069 & .0090 & .0143 & 6. 28 & 5.54 & 7. 03 & 8. 96 & 10.51 \\
\hline
\end{tabular}

TABLE 4. Mixing tests in the large flume

$($ Hydraulic radius $=3.27 \mathrm{~cm})$

\begin{tabular}{|c|c|c|c|c|c|c|c|c|c|}
\hline Run & $\rho_{1}$ & $\rho_{2}$ & $\nu_{1}$ & $\nu_{2}$ & $U_{c}$ & $U_{0}$ & $U_{1}$ & $U_{2}$ & $U_{3}$ \\
\hline & $\mathrm{g} / \mathrm{cm}^{3}$ & $\mathrm{~g} / \mathrm{cm}^{3}$ & $\mathrm{~cm}^{2} / \mathrm{sec}$ & $\mathrm{cm}^{2} / \mathrm{sec}$ & $\mathrm{cm} / \mathrm{sec}$ & $\mathrm{cm} / \mathrm{sec}$ & $\mathrm{cm} / \mathrm{sec}$ & $\mathrm{cm} / \mathrm{sec}$ & $\mathrm{cm} / \mathrm{sec}$ \\
\hline E-8 & 1.000 & 1. 0489 & 0.0090 & 0.0126 & 5.32 & 4.83 & 5. 92 & 7. 32 & 8. 33 \\
\hline E-9 & 1.000 & 1.046 & .0094 & .0131 & 5.57 & 4. 76 & 6.39 & 8.11 & 9.20 \\
\hline $\mathrm{E}-11$ & 1.000 & 1. 016 & .0093 & .0101 & 3.84 & 3.08 & 4. 65 & 6.16 & 6. 76 \\
\hline E-12 & 1.000 & 1. 150 & .0110 & .0404 & 8.27 & 7.02 & 9.52 & 12.50 & 13.45 \\
\hline E-13 & 1.000 & 1. 134 & .0103 & .0328 & 7.95 & 6. 58 & 9.32 & 11. 64 & 13.15 \\
\hline E-14 & 1.000 & 1. 116 & .0099 & .0251 & 7.13 & 5. 31 & 8.95 & 10.49 & 12. 41 \\
\hline $\mathrm{E}-15$ & 1.000 & 1. 110 & .0098 & .0213 & 7. 09 & 5. 79 & 8. 39 & 10.90 & 12. 45 \\
\hline E-17 & 1.000 & 1. 060 & .0106 & .0160 & 5. 50 & 5.00 & 6.00 & 6. 92 & 7. 98 \\
\hline E-18 & 1.000 & 1. 031 & .0100 & .0122 & 4. 93 & 4. 46 & 5. 20 & 6.86 & 7. 34 \\
\hline E-19. & 1.000 & 1. 033 & .0101 & .0125 & 4. 37 & 3.46 & 5. 28 & 6.17 & 7.03 \\
\hline $\mathrm{E}-20$ & 1.000 & 1.030 & .0101 & .0122 & 4. 36 & 3.92 & 4.80 & 6. 29 & 6. 96 \\
\hline $\mathrm{E}-21$ & 1.000 & 1. 022 & .0098 & .0112 & 4. 36 & 3.56 & 5.17 & 6.38 & 7. 55 \\
\hline
\end{tabular}

III. A Theoretical Basis for the Dimensionless Parameter of the Critical Velocity

If it were not for the inertia and the restraining effect of the upper current on the motion of the interfacial internal waves, we would introduce directly the criterion for wind-generated waves derived by Jeffreys as the desired criterion $[1,2] .{ }^{1}$ Jeffreys has shown that at the moment of developing wind waves

$$
U^{3}=\frac{27}{S} \nu_{2} g \frac{\Delta \rho}{\rho_{1}},
$$

where $U$ is the velocity of wind over water that is still initially, $\nu_{2}$ is the kinematic viscosity of water, $\Delta \rho$ is the difference between the densities of water and air, $\rho_{1}$ is the density of air, $S$ is a numerical constant, and $g$ is the acceleration of gravity.

Jeffrey's method for the analysis of the wind velocity criterion shows a marked departure from the well-known derivation due to Kelvin. The hypothesis that the flow in air is irrotational is abandoned, and instead it is supposed that the motion of air is such as to introduce a pressure variation over the water surface of the magnitude

$$
p=S \rho_{1} U^{2} d h / d x,
$$

where $S$ is a numerical constant referred to as the sheltering coefficient, $U$ is the velocity of the air over the crest, $h$ is the surface elevation of the wave above the undisturbed surface, and $x$ is in the direction of wind motion.

Jeffreys has concluded from observations on the initial formation of wind waves in a river and in the waters of a large pond that the velocity of a wind just strong enough to raise waves is 110 $\mathrm{cm} / \mathrm{sec}$, the average of three observations. The corresponding value of $S$ is 0.274 . Substituting these values in eq 1, we get for the criterion, writing

$$
\Theta=\left(\nu_{2} g \frac{\Delta \rho}{\rho_{1}}\right) / U
$$

the value

$$
\theta=0.215 \text {. }
$$

The problem of interfacial waves between two moving layers is a question of small oscillations

\footnotetext{
1 Figures in brackets indicate the literature references at the end of this paper.
} 
about a steady state of motion. Let the velocity of the upper layer be $U$, and of the lower layer zero. Let the corresponding densities be $\rho_{1}$ and $\rho_{2}\left(=\Delta \rho+\rho_{1}\right)$. The wavelength and the period being $\lambda$ and $T$, the internal waves may be assumed to be given by

$$
h=a \cos (k x-\sigma t) .
$$

where $k=2 \pi / \lambda$ and $\sigma=2 \pi / T$. Supposing that the motions are irrotational everywhere, that the wavelengths are small in comparison with the depths of the layers, and that the wave height is small with respect to the wavelength, analysis gives for the velocity $\omega$ of the waves

$$
\omega=\frac{\sigma}{k}=\frac{U}{2}+\left[\frac{1}{2} \frac{g}{k} \frac{\Delta \rho}{\rho_{1}}-\frac{1}{4} U^{2}\right]^{\frac{1}{2}},
$$

provided that the square of $\Delta \rho / \rho_{1}$ is negligible with respect to unity [3]. In stable waves $\sigma$ is real, and thus

$$
U^{2} \leq \frac{2 g}{k} \frac{\Delta \rho}{\rho_{1}} .
$$

As was noted previously, the internal interfacial waves just prior to the setting-in of mixing are regular in form and move progressively downstream without changing their form. The regularity and the permanency of form indicate that there is a mechanism by which the upper current is furnishing energy to the wave motion of the lower liquid just sufficient to counter the dissipation due to viscous effects. There are three conceivable methods of furnishing the energy. The energy supply may be effected through the normal stresses $p_{z z}$, or the tangential stresses $p_{x z}$, or by the combination of these stresses. Since for the ultimate purpose of the analysis, which is the derivation of dimensionless parameters, the selection is not important, it will be supposed that the transfer is made by the normal stresses $p_{z z}$, and the tangential stresses $p_{x z}$ vanish.

The dependence of $p_{z z}$ on the wave form must be known. The dissipation of waves through viscous forces has a direct bearing on the relation, and it is necessary that the ordinary equations of motion involving the viscosity terms be considered. These equations, neglecting the second-order terms, are

$$
\begin{gathered}
\frac{\partial u}{\partial t}=-\frac{1}{\rho} \frac{\partial p}{\partial x}+\nu \nabla^{2} u, \nabla^{2}=\frac{\partial^{2}}{\partial x^{2}}+\frac{\partial^{2}}{\partial z^{2}} \\
\frac{\partial w}{\partial t}=-\frac{1}{\rho} \frac{\partial p}{\partial z}-g+\nu \nabla^{2} w
\end{gathered}
$$

and

$$
\frac{\partial u}{\partial x}+\frac{\partial w}{\partial z}=0
$$

It is implied that $z$ is drawn vertically upwards. It will also be supposed that the origin is in the undisturbed level of the interface, and that $x$ is taken in the direction of wave motion. The quantities $u$ and $w$ denote the particle velocity components along the axes $x$ and $z$, respectively.

It has been shown by Basset [3], that the above set of equations is satisfied by

$$
u=-\frac{\partial \phi}{\partial x}-\frac{\partial \psi}{\partial z}, \quad w=-\frac{\partial \phi}{\partial z}+\frac{\partial \psi}{\partial x},
$$

and

$$
p / \rho=\frac{\partial \phi}{\partial t}-g z
$$

provided that

$$
\nabla^{2} \phi=0, \quad \frac{\partial \psi}{\partial t}=\nu \triangle^{2} \psi .
$$

Basset [3] and later Lamb [4] have applied these to the determination of the damping of the surface waves of a homogeneous layer. In reworking the solutions, we wish to find the condition that corresponds to no damping. It is to be noted that only in the event that $\psi$ becomes negligible may the flow be regarded as irrotational.

It seems that the above equations and the solution derived from them can be applied directly to the case of internal waves after resorting to the following artifice. The presence of the upper current is imagined to be evidenced in two ways only. First, the dynamical action of the current consists of the normal stresses $p_{z z}$. Second, the intensity of gravity in the lower liquid is reduced from $g$ to $g^{\prime}$, so that

$$
g^{\prime}=k \omega^{2}
$$

Accordingly, in the place of eq 12 we will write

$$
p / \rho_{2}=\frac{\partial \phi}{\partial t}-g^{\prime} z
$$


For the case of infinite depth and liquid at rest corresponding to the wave profile,

$$
h=a \cos (k x-\sigma t),
$$

correspond the solutions of eq 11:

and

$$
\phi=A e^{k z} e^{i k x+\alpha t},
$$

with

$$
\psi=C e^{m z} e^{i k x+\alpha t},
$$

$$
m^{2}=k^{2}+\alpha / \nu .
$$

If the case of cellular waves be excluded, $m$ denotes that root which has its real part positive. Thus,

and

$$
u=-\left(i k A e^{k z}+m C e^{m z}\right) e^{i k x+\alpha t},
$$

$$
w=-\left(k A e^{k z}-i k C e^{m z}\right) e^{i k x+\alpha t},
$$

In this solution we are supposing that there is no bodily movement of the lower layer as a whole, so that the surface kinematical condition is

$$
\partial h / \partial t=\omega, \quad z=0,
$$

and this leads to

$$
h=-\frac{k}{\alpha}(A-i C) e^{i k x+\alpha t} .
$$

By Stokes' rule, the relations between the stresses and the dilatations are

$$
p_{z z}=-p+2 \mu \frac{\partial w}{\partial x},
$$

and

$$
p_{x z}=\mu\left(\frac{\partial w}{\partial x}+\frac{\partial u}{\partial z}\right)
$$

As regards the normal stresses, it is appropriate to suppose, since a regular progression of waves is imagined, that

$$
p_{z z}=-\beta A e^{i k x+\alpha \ell} .
$$

It being the convention to regard pressures as negative tensions, $\beta$ is a positive quantity. The dynamical surface conditions, from eq 15, 19, 20, $22,23,24$, and 25 are

$\left(\alpha^{2}+2 \nu k^{2} \alpha+g^{\prime} k-\beta \alpha\right) A+i\left(g^{\prime} k+2 \nu k m \alpha\right) C=0$,

and

$$
2 i \nu k^{2} A+\left(\alpha+2 \nu k^{2}\right) C=0 .
$$

The elimination of the quantities $A$ and $C$ leads to $\alpha^{2}+4 \nu k^{2} \alpha+g^{\prime} k-\alpha \beta-2 \nu k^{2} \beta+4 \nu^{2} k^{4}-4 \nu^{2} k^{3} m=0$.

where $\nu$ is small, the terms containing its square may be neglected, and

$$
\alpha^{2}+4 \nu k^{2} \alpha+g^{\prime} k-\alpha \beta-2 \nu k^{2} \beta=0 .
$$

This may be written

$$
\alpha^{2}+4\left(\nu k^{2}-\beta / 4\right) \alpha=-g^{\prime} k+2 \nu k^{2} \beta .
$$

The condition that the waves are neither damped nor growing in height requires that

$$
\begin{aligned}
& \qquad \nu k^{2}-\beta / 4=0 ; \\
& \text { or, } \beta=4 \nu k^{2} . \\
& \text { Thus, eq } 30 \text { reduces to } \\
& \alpha^{2}=-g^{\prime} k+2 \nu k^{2} \beta,
\end{aligned}
$$

or

$$
\alpha^{2}=-g^{\prime} k+8 \nu^{2} k^{4},
$$

and due to the approximations assumed

$$
\alpha^{2}=-g^{\prime} k,
$$

or

$$
\alpha=-\sigma i,
$$

and substituting the latter in eq 22 , since $\sigma / k=\omega$

$$
h=\frac{1}{i \omega}(A-i C) e^{i k x-i \sigma t} .
$$

Equation 27 shows that the ratio $C / A$ is a small quantity when $\nu$ is small. Neglecting $C$,

$$
h=\frac{A}{i \omega} e^{i k x-i \sigma t} .
$$

Putting

$$
\begin{gathered}
A / i \omega=a, \\
h=a \cos (k x-\sigma t) .
\end{gathered}
$$

To this corresponds the surface pressure variation

$$
p_{z z}=4 \rho_{2} \nu k^{2} \omega a \sin (k x-\sigma t),
$$

and therefore the pressure is smaller on the forward face of a wave and greater on the backward face, as is to be expected. 
Here, following Jeffreys, we introduce the supposition that

$$
p_{z z}=S \rho_{1}(U-\omega)^{2} d h / d x,
$$

that is,

$$
p_{z z}=S \rho_{1}(U-\omega)^{2} k a \sin (k \chi-\sigma t) .
$$

The implication is that for the maintenance and for the growth of waves $U$ is larger than $\omega$; that is, the current velocity is larger than $\omega$. When $U$ is smaller than $\omega$, the waves are damped. The latter statement is due to Kelvin [5].

Comparing eq 33 and 35 with each other and ignoring the small term involving $\Delta \rho / \rho_{1}$, and writing $\nu_{2}$ for $\nu_{1}$

$$
\frac{(U-\omega)^{2}}{\omega}=\frac{4 \nu_{2} k}{S} .
$$

This is the relation to be satisfied between the quantities $\omega, U$, and $k$ at the instant that the internal waves are about to increase in height. Returning to the inequality, eq 7 , it may be supposed that the wavelengths have reached the greatest values consistent with stability; that is

$$
k=\frac{2 g}{U^{2}} \frac{\Delta \rho}{\rho_{1}} .
$$

Eliminating $k$ between the latter two equations,

$$
U^{2}(U-\omega)^{2} / \omega=\frac{8}{S} \nu_{2} g \Delta \rho / \rho_{1},
$$

and since under these conditions

$$
\omega=U / 2,
$$

(see eq 6) we finally obtain

$$
U^{3}=\frac{16}{S} \nu_{2} g \Delta \rho / \rho_{1} .
$$

This may be written as

$$
\Theta=\left(\nu_{2} g \Delta \rho / \rho_{1}\right)^{1 / 3} / U,
$$

and is the desired form for the criterion of mixing.

\section{Experimental Value of the Criterion of Mixing}

To generalize the result of the theoretical analysis made above and in particular to examine the effect of the Reynolds number of the current on mixing, the data of the tests will be considered in the parametric forms

and

$$
\Theta=\left(\nu_{2} g / \Delta \rho / \rho_{1}\right)^{1 / 3}
$$

$$
\Theta=\Theta\left(U_{c} R / \nu_{1}\right) .
$$

In these, $\rho_{1}$ is the density of the upper flowing liquid, $\nu_{1}$ and $\nu_{2}$ are the kinematic viscosities of the upper and the lower liquids, respectively, $\Delta \rho$ is the difference in the densities of the two liquids, $U_{c}$ is the critical velocity of mixing, and $R$ is the hydraulic radius of the cross section of the flowing current.

However, prior to the consideration of the magnitude of the criterion it will be helpful to present the evidence of the effect of kinematic viscosity on the criterion itself. The criterion contains the quantities $\nu_{2}$ and $\Delta \rho / \rho_{1}$ each raised to its one-third power. The effect of the viscosity will be seen best and also most convincingly if a series of tests are conducted keeping $\Delta \rho / \rho_{1}$ constant or nearly constant and varying the kinematic viscosity $\nu_{2}$ over a large range. Most of the runs of the $D$ series in the medium flume fulfill this condition. The test data are given in table 2. The plotting shown in figure 4 is from this table. The critical

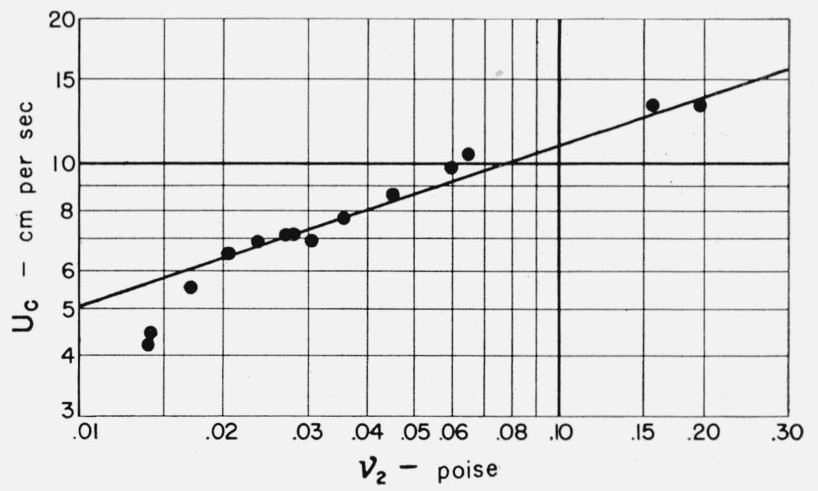

Figure 4. Relation of critical velocity of mixing to kinematic viscosity of heavy liquid.

velocity of mixing is treated as a function of the kinematic viscosity of the lower liquid. The great majority of the points aline themselves along a straight line having an inclination of one in three. Since the plotting is done logarithmically, this at least confirms the requirement of the theory.

As regards the criterion and the effect of Reynolds number on it, the values of the parameter 
$\Theta$ from all the tests are shown in figure 5. One glance at the figure suggests that the criterion has one constant value below a Reynolds number of 450 and another constant value above it:

and

$$
\Theta=0.127, R_{e}<450 ;
$$

$$
\Theta=0.178, R_{e}>450 .
$$

Significantly, $R_{e}=450$ is also the Reynolds criterion that separates the regime of turbulent flow from the regime of laminar flow.

Considering the distribution of the $\theta$ points more carefully for Reynolds numbers above 450, some systematic variations from the mean value, 0.178 , are detectable. This would mean that there are other factors having a secondary influence on the value of the mixing criterion. Such factors

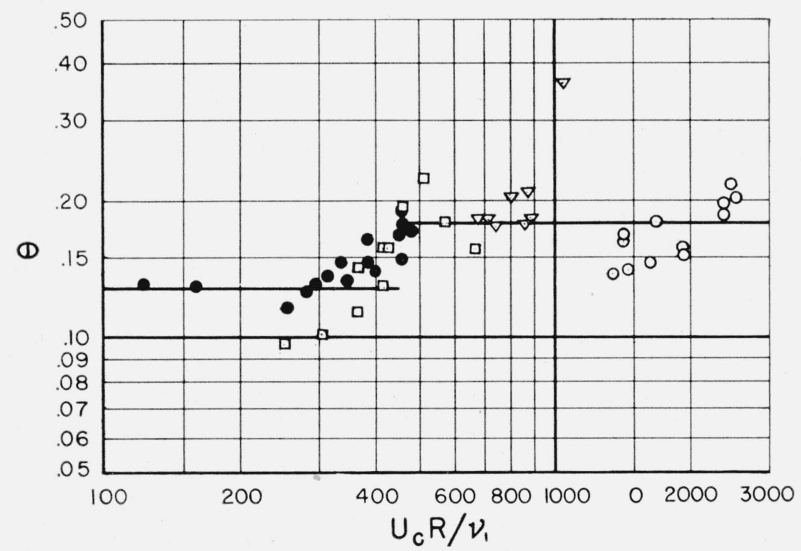

FIGURE 5. Relation of criterion of mixing to the Reynolds number of the current of light liquid.

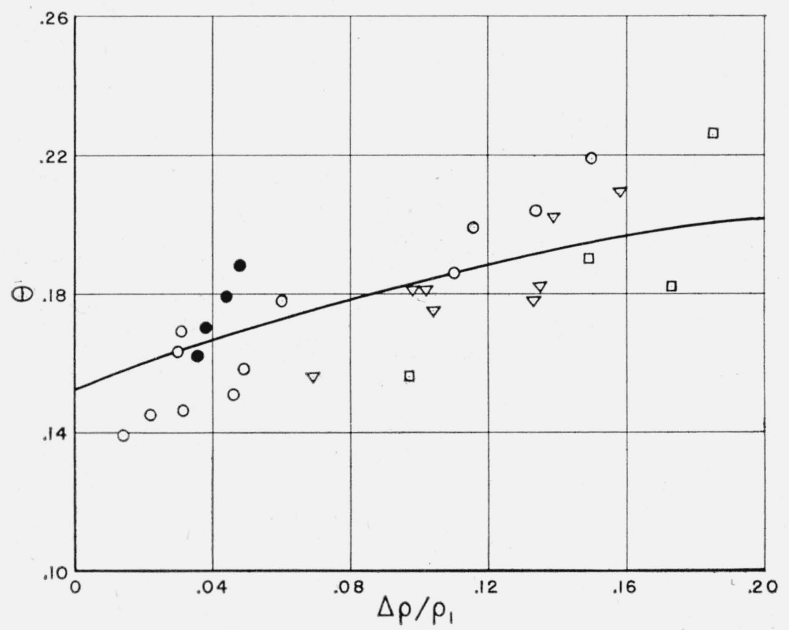

Figure 6. Relation of criterion of mixing to the relative densities of the two liquids. may very well be (a) the surface tension between the two liquids, (b) the difference between the kinematic viscosities of the two liquids, and (c) the relative densities of the two liquids. It would be a futile effort to speculate on the effect of these factors separately. Since in these present tests the solutions were prepared from the same solvent and solute, one may say that all these factors or quantities are functions of $\Delta \rho / \rho_{1}$. Thus it would be appropriate and also sufficient to consider the dependence of $\theta$ on $\Delta \rho / \rho_{1}$. For that purpose the graph in figure 6 has been prepared. That $\theta$ values vary with $\Delta \rho / \rho_{1}$ appears to be unmistakable.

It is interesting to compare the value of the criterion for the mixing between two liquids obtained in these tests with the criterion that may be associated with the formation of wind waves. The latter, as determined from the observations of Jeffreys, has been mentioned previously. The value found by Jeffreys was 0.215 , and this is not very different from the mean value of the mixing criterion of the tests; that is, the value 0.178 . When the very great differences in the conditions of these two phenomena are considered, the closeness of the two values is quite surprising.

\section{Mean Velocity Distribution in Currents}

The fact, as shown earlier in this paper, that the criterion of mixing takes on two different values, one value when the Reynolds number of the current is below 450 and another value when above, may be explained on the grounds of velocity distributions. For the changes of the interface, the velocities near the interface are the important ones. The representation of the data, however, was based on the mean flow, and it will be illuminating to examine the variation of the mean velocity distributions in the cross section of a square channel for laminar and turbulent flows.

For this purpose we take a square of side $2 l$, draw an inside square of side $2(l-s)$, with the centers coinciding, and consider the space between the two (fig. 7). This space is a frame of width s. Denoting the mean velocity in the complete large square by $\bar{u}$, and in the frame only by $\bar{u}_{s}$, we wish to find the variation of $\bar{u}_{s} \sqrt{u}$ with $s / l$. If the flow is turbulent, using the Blasius law of velocity, and supposing that the wall shear is constant along the entire perimeter, it is a simple matter to show that 


$$
\frac{\bar{u}_{s}}{\bar{u}}=\frac{(s / l)^{\frac{1}{4}}(15-8 s / l)}{7(2-s / l)} .
$$

Values of this relation are shown in figure 7 . Accordingly, when the flow is turbulent, the velocities near the interface are not much different from the mean velocity of the entire section. In the actual conditions of the tests, the differences are somewhat smaller than is indicated by the curve, since the liquid at the interface is in motion, and this was ignored in the derivation given above.

The computation of a similar result for the condition of laminar flow could have been made from

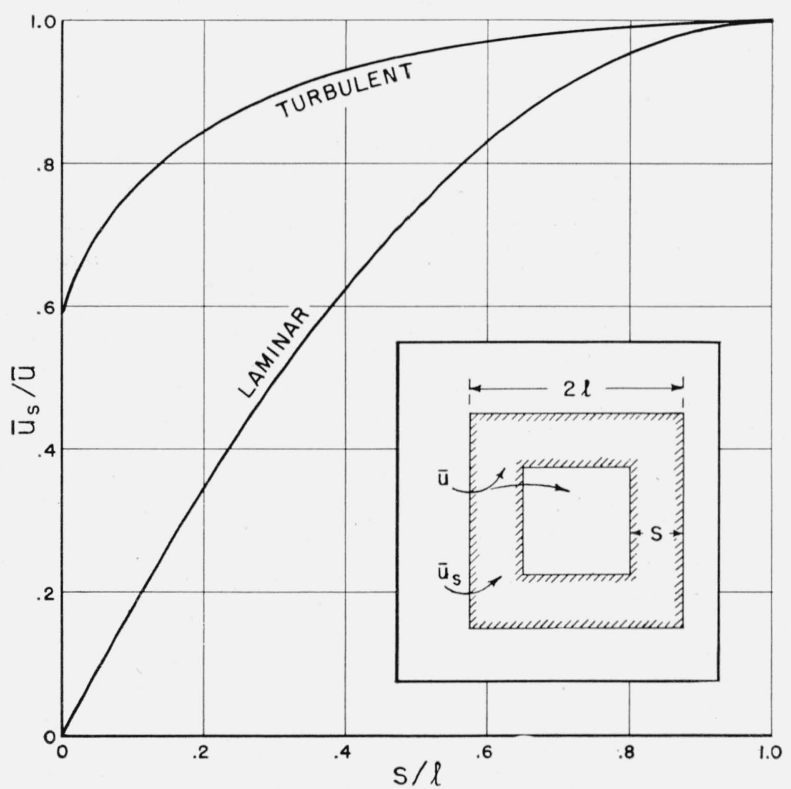

Figure 7. Mean velocity distributions in a square channel for laminar and turbulent regimes.

the formula of velocity distribution in a rectangular channel that Bateman gives [6]. However, computations from this particular formula are somewhat cumbersome, and it was decided to derive a simpler formula involving algebraic polynomials only.

Taking the axis of the channel as the $z$ axis, pressures decreasing with $z$, the equation of motion is

$$
\frac{\partial p}{\partial z}=\mu\left(\frac{\partial^{2} u}{\partial x^{2}}+\frac{\partial^{2} u}{\partial z^{2}}\right)
$$

where $p$ is the pressure, uniform across a normal section. Let $2 l$ be the length of the square side. Let $u_{m}$ be the maximum velocity in the section; that is, the velocity at $x=0, y=0$. Writing the variables

$$
\left.\begin{array}{c}
\frac{u}{u_{m}}=\chi, \\
\frac{x}{l}=\zeta, \\
\frac{y}{l}=\eta,
\end{array}\right\}
$$

and introducing the dimensionless form of the pressure term,

$$
\frac{1}{\mu} \frac{l^{2}}{u_{m}} \frac{\partial p}{\partial z}=-\kappa
$$

the equation of motion now becomes

where

$$
\nabla^{2} \chi=+\kappa,
$$

$$
\nabla^{2}=\frac{\partial^{2}}{\partial \xi^{2}}+\frac{\partial^{2}}{\partial \eta^{2}}
$$

The boundary conditions are

$$
\left.\begin{array}{lcc}
\chi=0, & \xi=1, & -1 \leq \eta \leq 1, \\
\chi=0, & \eta=1, & -1 \leq \xi \leq 1, \\
\chi=0, & \xi=0, & \eta=0 .
\end{array}\right\}
$$

The velocity term may be expanded in terms of algebraic polynomials:

$$
\chi=1+a \chi_{2}+b \chi_{4}+c \chi_{8}+\ldots .
$$

where $a \chi_{2}$ is a particular solution of eq 49 , and the remaining polynomials $\chi_{4}, \chi_{8}, \ldots$ are the solution of

$$
\nabla^{2} \chi=0
$$

The quantities $a, b, c$ are numerical constants.

Since the cross section is a symmetrical one, the polynomials $\chi_{2}, \chi_{4}, \chi_{8} \ldots$ are functions of $\xi^{2}$ and $\eta^{2}$. Also, in particular, the section is a square, and thus the value of the polynomials will not be altered when $\zeta$ is replaced by $\eta$ and $\eta$ is replaced by $\zeta$. Subject to these conditions

$$
\left.\begin{array}{l}
\chi_{2}=\zeta^{2}+\eta^{2}, \\
\chi_{4}=\zeta^{4}+\eta^{4}-6 \zeta^{2} \eta^{2}, \\
\chi_{8}=\zeta^{8}+\eta^{8}-28 \zeta^{6} \eta^{2}-28 \zeta^{2} \eta^{6}+70 \zeta^{4} \eta^{4} \ldots
\end{array}\right\}
$$

As $a \chi_{2}$ is solution of eq 50 :

$$
a=-\kappa / 4 \text {. }
$$


This determines $\kappa$ from the constant $a$. For the determination of the constants $a, b$, and $c$ we write from eq 51

$$
\left.\begin{array}{ccc}
\chi=0, & \eta=1, & \zeta=0, \\
\chi=0, & \eta=1, & \zeta=1, \\
\xi^{2}=0, & \eta=1, & \zeta=0 .
\end{array}\right\}
$$

Accordingly,

$$
\left.\begin{array}{l}
a+b+c=-1, \\
2 a-4 b+16 c=-1, \\
a-6 b-28 c=0 .
\end{array}\right\}
$$

Solving these simultaneously,

$$
\left.\begin{array}{l}
a=-0.8456, \\
b=-0.1581 \\
c=-0.00368 .
\end{array}\right\}
$$

The relation between the pressure fall and the maximum velocity now is

$$
\frac{\partial p}{\partial z}=3.382 \frac{\mu u_{m}}{l^{2}},
$$

and between ${ }^{-}$the mean velocity and maximum velocity,

$$
\bar{u}=0.4797 u_{m} .
$$

The values of $\bar{u}_{s} /{ }^{u}$ as determined in this analysis are shown likewiseiin figure 7 by the curve labeled "laminar." Acc ,rdngly, in laminar flows the velocities near the interface are appreciably lower than the mean velocity of the entire section, and this fact will lead to a smaller value of the mixing criterion if it is computed on the basis of mean flow.

\section{Nature of Assumptions in the Analysis for the Criterion of Mixing}

It is necessary to call attention to the approximate nature of the assumptions that were made in the derivation of the criterion.

The first assumption is that the velocity of advance of the internal waves can be obtained by the ordinary well-known wave formula. The formula refers to the case of discrete densities and a surface of velocity discontinuity at the interface. Actually, although the condition of discrete velocities on the two sides of the interface is nearly satisfied, on the contrary the velocities are not discontinuous. In fact, at the instant just preceding the formation of nondeformable waves, the flow at the interface and on the two sides of it is viscous, and the regime is laminar. According to an analysis relative to the boundary layer of the interface when the density difference and the viscosity difference of the two liquids are small, the velocity at the interface is nearly half that of the current, the lower liquid being a pool [7]. During some of the runs small wax beads loaded with lead particles were introduced. These would fall through the lighter liquid, but in reaching the lower liquid the beads would float at the interface. The motion of the beads, whenever this was observed, showed that the interfacial velocity was nearly half that of the current. For example, in run $\mathrm{E}-19$, corresponding to the current velocities $2.29,3.53$, and $4.56 \mathrm{~cm} / \mathrm{sec}$, the interface velocities noted were $1.37,1.78$, and $2.12 \mathrm{~cm} / \mathrm{sec}$, respectively. Thus, in a more precise derivation of the velocities of the interface waves, attention might be given to the laminar regime of the interface.

The second assumption consists of a statement as to the wavelengths attained at the instant the mixing is about to commence. The wavelengths for the current velocity are maximum values consistent with the condition of stability; that is,

$$
\lambda=\frac{\pi U_{c}^{2}}{g \Delta \rho / \rho_{1}} .
$$

Whenever records of wavelengths were kept, the recorded values appeared to be in agreement with the formula, as can be seen from the data in figure 8. The wavelengths were estimated rather than accurately measured. This fact and also the fact that the measurements are few appreciably weaken the value of the evidence shown.

The third assumption assigns to the waves a value of velocity propagation in conformity with the wavelengths; that is,

$$
\omega=U_{c} / 2 .
$$

Although in many cases this relation held nearly true, there were noted instances when the wave velocity was larger, and in the vicinity of $\omega=0.8 U_{c}$.

The final assumption is in regard to the supply of energy to the internal waves. The source of supply is thought to be due to the eddies in the 


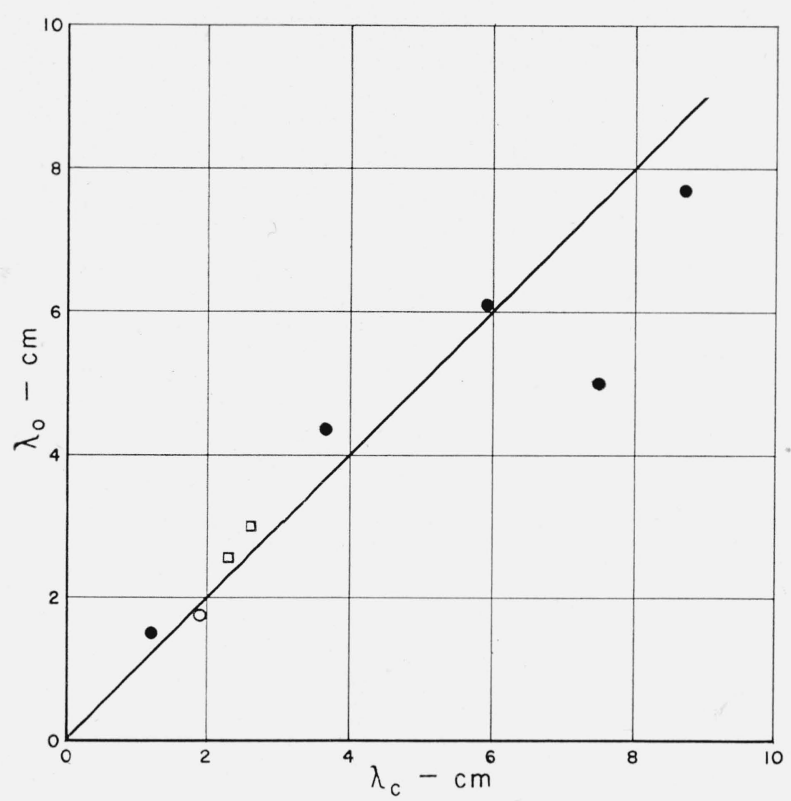

Figure 8. Comparision of observed with computed wavelengths at initial mixing.

troughs or depressions of the upper current. The supply is of such magnitude as to balance that part of the dissipation of the internal waves taking place in the liquid of the lower pool. Probably this idea is correct. However, our attempts to verify it by observations on the mechanism of flow did not prove successful.

Whatever be the details of a new analysis, carried out with greater rigor and with proper assumptions, the parametric form of the criterion of mixing will not be modified. Whatever changes are obtained will be in the nature of replacing the numerical constants appearing in eq 40, either by new constants or by a new function involving the densities and viscosities of the two liquids appearing as a correction.

\section{The Amount of Mixing With Increasing Current Velocity}

The second purpose of the investigation was to determine the amount of mixing when the velocities of the current are above the critical value. The amount of mixing, $q$, may be defined as the volume of the heavier liquid that crosses unit area of the interface in unit time. The dimensions of the quantity $q$ are that of velocity, and the symbol $U_{m}$ will be used for this quantity. The tests of the amount of mixing were made with the large flume.
The experimental determinations of $q$, or of $U_{m}$, are based on the following simple concepts. Let $V$ be the volume of the upper liquid. In these tests, $V$ is the volume of the lighter liquid contained initially in the reservoir below and prior to its introduction into the flume. The heavy liquid of the pool is highly colored with a darkish-blue water-soluble dye. Let $N$ be the number of coloring particles in unit volume of the heavier liquid. Let $A_{i}$ be the total area of the interface. As a result of mixing the upper liquid becomes tinted. Let $d N$ be the number of coloring particles entering into unit volume of the upper liquid during the time interval $\Delta t$ when the current velocity is constant for this interval.

Because of continuity of mass

$$
V d N=A_{i} q N \Delta t .
$$

Introducing the symbol

$$
\begin{gathered}
\Delta C=d N / N, \\
q=\frac{V \Delta C}{A_{i} \Delta t} .
\end{gathered}
$$

We may refer to $\Delta C$ as the increase in the fractional concentration of the upper liquid.

During the conduct of tests the velocity of the upper current was changed to the values $u_{1}, u_{2}$, $u_{3}$, . . at the times $t_{0}, t_{1}, t_{2}$, . . . Thus, during the time interval $t_{1}-t_{0}$, the velocity of the current was constant and equal to $u_{1}$. In general, during the time interval $t_{n}-t_{n-1}$, the current velocity was $u_{n}$. At the times $t_{0}, t_{1}, t_{2}, \ldots$. . ; that is, at the times that new velocities were established, samples $s_{0}, s_{1}, s_{2}, \ldots . s_{n}$ of the liquid in the reservoir below were taken. Let the partial concentrations of these samples be $C_{0}, C_{1}, C_{2}$,

$C_{n}$, respectively. Thus to obtain the rate of mixing $q_{n}$, when the velocity of the current is $u_{n}$, we consider the equation

$$
q_{n}=\frac{V\left(C_{n}-C_{n-1}\right)}{A_{i}\left(t_{n}-t_{n-1}\right)} .
$$

The determination of the partial concentrations $C$ of the samples $s$ was effected by the use of photoelectric cells. Standard solutions of known partial concentrations of $0,2,4,6,9$, and 10 percent, using the heavier liquid from the pool as base, were prepared. These were introduced one 
at a time into a rectangular glass jar with plane polished surfaces. The jar was interposed between a source of light of constant illuminationintensity and a photoelectric cell, and the milliampere reading of the cell excitation was noted. The milliampere reading was taken as a measure of the partial concentration of the sample in the jar. These measurements of the standard solutions of known partial concentrations established a calibration curve. Next, the milliampere readings of the samples $s_{0}, s_{1}, \ldots s_{n}$ were obtained. By reference to the calibration curve, the partial concentrations $C_{0}, C_{1}, \ldots C_{n}$ were then determined.

The mixing-rate values as determined by the above process for run $\mathrm{E}-14$ are given in figure 9 . The circles correspond to the photoelectric measurements. The square marks the velocity $U_{1}$, which by visual observation was associated with the condition of slight mixing. The triangle marks what we have judged as the critical velocity, $U_{c}$. The distribution of the observation points marked by circles can be best approximated by a curved line. This same condition was observed in practically all of the runs of the $\mathrm{E}$ series. Nevertheless, for the purpose of effecting simplicity in the representation, a straight line is drawn, starting from the point of slight mixing, $U_{1}$. This means that, approximately, the amount of mixing is proportional to the increase of the current velocity above the velocity $U_{1}$ of slight mixing. Accordingly,

$$
U_{m} /\left(U-U_{1}\right)=C \text {. }
$$

The value of $C$, the constant of proportionality, from all the runs of the series $\mathrm{E}$ tests, made with the large flume, are shown in figure 10. It would seem that the magnitude of the constant decreases when the relative density of the pool liquid is increased. We shall ignore, provisionally, the effect of density. Furthermore, there is a correlation between the velocities $U_{1}$ and $U_{c}$, such that, $U_{1}=1.15 U_{c}$. On this basis, the simplified form of the law of mixing rate will be

$$
U_{m}=3.5 \times 10^{-4}\left(U-1.15 U_{c}\right) .
$$

It was unfortunate that, due to the limitations of the apparatus and the capacity of the pump, it was not possible to have current velocities higher

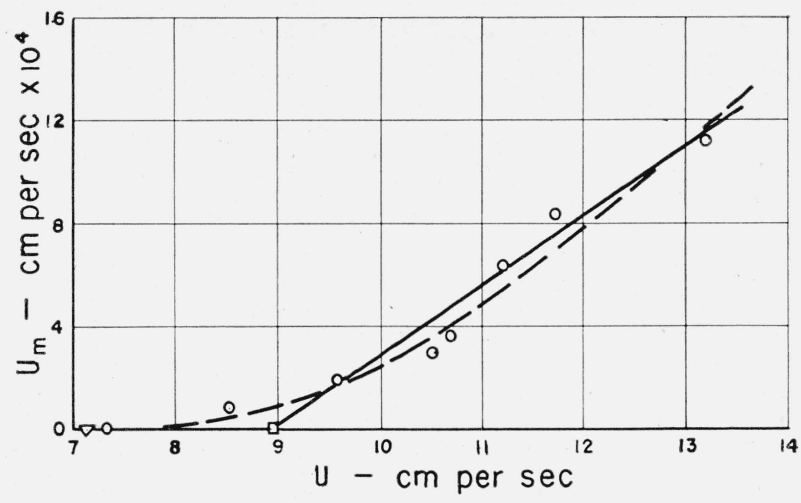

Figure 9. Relation of amount of mixing to velocities of the current of light liquid.

than those employed in the tests. It is important to have an idea of the limiting velocity where the law ceases to be linear, or nearly linear, and of the marked changes that may occur at the higher velocities. Another undesirable aspect of the

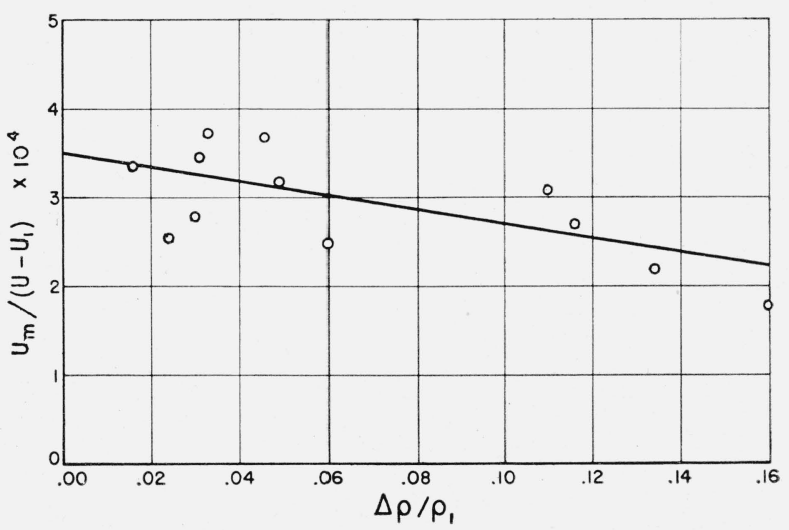

Figure 10. Relation of amount of mixing to relative densities of the two liquids.

apparatus was the shortness of the flumes. It was not always possible to divert correctly the dragging of heavier liquid at the exit end. In some cases the diversions were somewhat excessive and in others somewhat deficient, and, therefore, because of the shortness of the flume, errors were made in estimating the mixing rates, $U_{m}$.

It will be interesting at this point to inquire about the increased frictional force between the two liquids due to the mixing. The frictional force caused by the maintenance of the waves at the interface may be expressed as

$$
\tau_{1}=\frac{1}{U} \frac{d E}{d t}
$$


where $U$ is the current velocity and $d E / d t$ the dissipation in the waves per unit area of interface. Writing for the shear,

$$
\tau_{1}=f_{1} \rho U^{2} / 2,
$$

where $f_{1}$ is the coefficient of friction, it is seen that

$$
f_{1}=\frac{2}{\rho U^{3}} \frac{d E}{d t} .
$$

As an estimate it may be supposed that the dissipation will take on the same value as for surface waves in contact with air. The expression is

$$
\frac{d E}{d t}=16 \pi^{3} \mu \omega^{2} a^{2} / \lambda^{3}
$$

Hence,

$$
f_{1}=32 \pi^{3} \frac{\nu}{\lambda U}\left(\frac{\omega}{U}\right)^{2}\left(\frac{a}{\lambda}\right)^{2}
$$

Assume that $\omega=U / 2$, a condition that was observed in some tests, and

$$
f_{1}=8 \pi^{3}(\nu / \lambda U)(a / \lambda)^{2} .
$$

This would indicate that, $a$ and $\lambda$ remaining the same, $f_{1}$ decreases with increasing $U$. Actually, however, $a$ increases with $U$. Let it be assumed for the present that $a^{2}$ is proportional to $U$ and that $\lambda$ is unaltered. Then the value of the coefficient can be obtained by noting the conditions at the onset of mixing, and

$$
f_{1}=8 \pi^{3} \frac{\nu}{\lambda U_{c}}\left(\frac{a}{\lambda}\right)^{2},
$$

where $a$ and $\lambda$ are the wave dimensions at the condition of slight mixing. Using the observed values $\lambda=2.0 \mathrm{~cm}, a=0.2 \mathrm{~cm}, \nu=0.01 \mathrm{~cm}^{2} / \mathrm{sec}$, and $U_{c}=10 \mathrm{~cm} / \mathrm{sec}$, we obtain

$$
f_{1}=0.0010 \text {. }
$$

Denoting next the additional shearing resistance due to mixing by $\tau_{2}$,

$$
\tau_{2}=\rho U_{m} U,
$$

since the liquid crossing the interface is initially at rest, and after crossing the interface it takes on the velocity of the current. Writing $\tau_{2}$ in the form

then

$$
\tau_{2}=f_{2} \rho U^{2} / 2,
$$

$$
f_{2}=2 U_{m} / U \text {. }
$$

Introducing the expression for $U_{m}$ from eq 68

$$
f_{2}=7.0 \times 10^{-4}\left(1-1.15 U_{c} / U\right) .
$$

Thus, if the mixing law as found above is valid also for large values of $U$, the coefficient of friction due to mixing is

$$
f_{2}<0.0007 \text {. }
$$

These results show that for moderately small channels and moderately small velocities the friction coefficient at the interface is smaller than the coefficient ordinarily obtained for smoothwalled channels. Perhaps this explains our failure to obtain reliable readings of pressure fall along the flumes during the tests.

\section{Mixing in a Large Body of Water}

The bearing of the results of the present investigation on the interfacial mixing occurring in large bodies of water in stratified flow must be discussed.

In the laboratory experiments, where relatively short flumes are used and one of the liquids, the lower or the upper, is at rest, and the other is in motion, the state of the interface is one of discontinuity of density. In the case when the lower heavy liquid moves, it will be supposed that the flow has continued for a long time and that the characteristic wave front is absent. Under these conditions, the interfacial stability, the critical velocity of mixing, and the mixing for velocities above the critical may be studied. All these, however, refer to a reach that is to be looked upon as a type of initial length.

In the phenomenon transpiring in natural environments and thus involving large bodies of water in stratified flow, it may be assumed that conditions arise so that initial reaches are established. It is expected that the changes taking place in the initial length will be similar to those observed in a laboratory. What is not known definitely in this respect is the direct applicability of the results to be obtained in a laboratory investigation to the prototype magnitudes. Certainly, however, a qualitative similarity, at least, must exist.

If that is granted, the application of the laboratory results must be restricted to a very short reach, which will be viewed as the initial reach. 
Beyond this in the remaining reach, which will be of considerable length, the conditions for mixing and the manner of mixing will be of a different type, obeying different laws. For the mixing in the initial reach will establish in the following adjoining reach a transition layer between the liquids, and in this transition layer the density will vary continuously. As an illustration of how this can be brought about, we may visualize the following situation. A current of fresh water of depth $H$ is flowing with a uniform velocity over a pool of heavier liquid. In places in the initial length, portions of liquid coming from the lower pool will spread themselves in the upper current only gradually. Let it be supposed that the spreading is proportional to time, this being measured from the instant of departure from the lower liquid. The density gradient established for this case can be obtained readily. Taking the instant when the spreading is completed and the area covered is a square of sides of length $H$, the concentration along a vertical may be represented by

$$
C=A+B / y,
$$

for finite distances away from the interface.

Now, the actual law of spreading for a given actual case may not be as simple as in the above illustration. As long as it is assumed that spreading of liquids ejected from below into the upper current and in the initial length is gradual, a qualitatively similar law for the concentrations as the one mentioned above will be expected. But these distributions imply the existence of transi- tion layers. Whereas the mixing in the case of sharp interfaces is brought about by the ejection of eddies at the crests of the internal waves, the mixing through the transition layers must be associated with the momentum exchange of turbulent motion. This is a matter to be approached using the basic ideas of the Prandtl and Richards criterion for mixing and is, therefore, a subject outside the scope of the present investigation.

The author expresses his appreciation to $\mathrm{K}$. Hilding Beij, Assistant Chief of the National Hydraulic Laboratory, for his editorial review of the paper and for constructive suggestions.

\section{References}

[1] Harold Jeffreys, On the formation of water waves by wind, Proc. Roy. Soc. [A] 107, 189 (1925).

[2] Harold Jeffreys, Formation of water waves by wind, Proc. Roy. Soc. [A] 110, 241 (1926).

[3] A. B. Basset, A treatise on hydrodynamics, Vol. II (Cambridge University Press, Cambridge, Eng. 1888).

[4] H. Lamb, Hydrodynamics (ed. 5), (Cambridge University Press, Cambridge, Eng., 1924).

[5] Lord Kelvin, Baltimore lectures on molecular dynamics and wave theory of light (Cambridge University Press, Cambridge, Eng., 1904).

[6] H. L. Dryden, F. D. Murnaghan, H. Bateman, Report of the committee on hydrodynamics, Bull. Nationa! Research Council, No. 84 (1922).

[7] G. H. Keulegan, Laminar flow at the interface of two liquids, J. Research NBS 32, 303 (1944), RP1591.

Washington, May 26, 1949. 